\title{
Learnability of complex phonological interactions: an artificial language learning experiment
}

\author{
K. Michael Brooks ${ }^{*}$, Bozena Pajak ${ }^{\dagger}$, Eric Baković* \\ *University of California, San Diego; ${ }^{\dagger}$ University of Rochester
}

1. Introduction. Natural languages exhibit evidence that phonological processes interact with one another in complex ways: the application or applicability of one process has the potential to impact that of another. These interactions come in various forms, including simple interactions such as feeding, in which the application of one process enhances the applicability of another, and bleeding, in which the application of one process diminishes the applicability of another.

A more complex example comes from Baković's (2005) analysis of the alternations in the English past tense, as shown in (1). In this analysis, a vowel is epenthesized to avoid a sequence of identical or near-identical consonants, as in (1c) and (1d). Crucially, vowel epenthesis in the case of near-identical consonants in (1d) is driven by an interaction between voicing assimilation and avoidance of identical consonants: with no epenthesis in a form such as /weit/ $+/ d /$, the independent voicing assimilation process in (1b) would otherwise produce an illegal sequence of identical consonants (*[wertt]).
(1) a. Basic form
b. Voicing assimilation
$/ \mathrm{wæg} /+/ \mathrm{d} / \rightarrow[$ wægd $]$
$/ \mathrm{wak} /+/ \mathrm{d} / \rightarrow[\mathrm{wakt}]$
'wagged'
c. Epenthesis between identical Cs
$/$ werd $/+/ \mathrm{d} / \rightarrow$ [weIdəd]
'walked'
'waded'
d. Interaction preventing identity
$/$ wert $/+/ \mathrm{d} / \rightarrow[$ wertəd $] \quad(*[$ wertt $])$
'waited'

As with any other aspect of a language, these interactions can only be learned from their manifestations in the surface forms of the language. However, the way that they are learned remains a question. It may be the case that interactions between processes must be directly observed to be learned; in terms of the example above, English learners would have to encounter forms such as 'waited' in order to learn that epenthesis applies due to the applicability of assimilation. On the other hand, it may be possible that these learners are capable of inferring the interaction between epenthesis and assimilation simply by observing the processes occurring independently. That is, forms such as 'waded', which shows insertion between identical consonants, and 'walked', which shows assimilation, together provide sufficient evidence for forms like 'waited', in which assimilation would lead to identity but can be prevented by epenthesis.

2. Experiment. To shed light on how phonological interactions are learned, we constructed an artificial language containing processes similar to those in English described above, with the potential to interact in a similar way. Critically, we withheld any direct evidence that our processes could interact, leaving only indirect evidence in the independent existence of the processes involved. In this way, we can answer the question as to whether direct evidence is necessary in the learning of phonological interactions.

Participants were told that they would be learning to form compound words in a language that they do not speak, and that these compounds are all composed of words for objects and their properties. There was a total of 18 objects and 31 properties. The words were presented auditorily, pictorially, and orthographically, as in (2), in the following order: first the object (' jod'), then the property ('dit'), then the compound of the two ('jodadit'). ${ }^{1}$ Crucially, forming a compound frequently involved the application of one of several phonological processes at the compound juncture, as discussed in detail below.

(2)
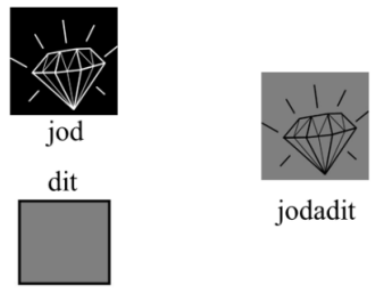

\footnotetext{
${ }^{1}$ Orthographic representations were consistent with the IPA, except where it conflicted with English orthography. This was the case for [J], [3], and [j], which were represented as $s h, j$, and $y$, respectively.
} 
Participants exposed to the Assimilation Language were presented with combinations of words showing either no change at the compound juncture (3a) or a change involving one of three phonological processes $(3 a-d)$.

\begin{tabular}{|c|c|c|c|}
\hline (3) a. Basic form & no change & & $/ \mathrm{lom} /+/$ tol $/ \rightarrow[$ lomtol $]$ \\
\hline b. Epenthesis between identical Cs & $\varnothing \rightarrow \mathrm{a} / \mathrm{C}_{i}$ & $\mathrm{C}_{i}$ & $/$ lot $/+$ tol $/ \rightarrow[$ lotatol $]$ \\
\hline c. Place assimilation & $\int, 3 \rightarrow \mathrm{s}, \mathrm{z} /$ & alveolar & $/ \mathrm{lo} f /+/$ tol $/ \rightarrow[$ lostol $]$ \\
\hline d. Lateralization filler & $r \rightarrow 1 /$ & & $/$ lor $/+/$ tol $/ \rightarrow[$ loltol $]$ \\
\hline
\end{tabular}

The items in (3b) and (3c) are roughly analogous to the epenthesis and voicing assimilation seen in the English past tense. Here, a potential for interaction occurs in $\mathrm{CV} f+\mathrm{sVC}$ and $\mathrm{CV}_{3}+\mathrm{zVC}$ forms (e.g., $/ \mathrm{lo} / \mathrm{t} / \mathrm{sol} /)$, in which assimilation would lead to a sequence of identical consonants ([lossol]), but epenthesis could occur to prevent it ([lofasol]). The forms in which interaction is possible were crucially withheld from training. Therefore, if indirect evidence is sufficient to infer interaction, we predict that participants would consider compounds such as [lofasol] to be grammatical in this language.

As a control, we constructed a minimally different Labialization Language, in which place assimilation $(3 \mathrm{c})$ was replaced by a labialization process $(4 \mathrm{c})$.

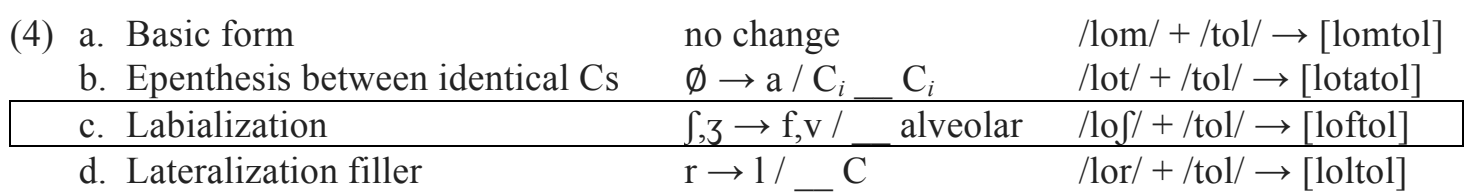

Unlike in the Assimilation Language, in the Labialization Language there is no possibility for epenthesis to interact with another process. Consider the $/ \mathrm{lo} / \mathrm{H} / \mathrm{sol} /$ example again. Labialization before alveolars will never create [ff], [ss], or any other sequence of identical consonants. As a consequence, the withheld $\mathrm{CVJ}+\mathrm{sVC}$ and $\mathrm{CV}_{3}+\mathrm{zVC}$ sequences should result in straightforward applications of the labialization process: $/ \mathrm{lo} / \mathrm{H} / \mathrm{sol} /$ should simply become [lofsol]. Therefore, participants trained on the Labialization Language should consider epenthetic compounds such as [lofasol] to be ungrammatical in this language.

Participants ( $\mathrm{n}=50,25$ per language) were monolingual native English speaking undergraduate students at the University of California, San Diego. They were trained on either the Assimilation Language or the Labialization Language. The training consisted of 2 blocks of 64 trials each. Each block contained trials of the following types (16 trials each): no change ((3a) and (4a)), filler ((3d) and (4d)), epenthesis $((3 b)$ and (4b)), and either assimilation (3c) or labialization (4c), depending on the language. Critical $\mathrm{CVJ}+\mathrm{sVC}$ and $\mathrm{CV}_{3}+\mathrm{zVC}$ items were withheld from both languages.

Testing consisted of a well-formedness judgment task on a five-point scale. Test compounds were composed using all of the objects and properties used in training, as well as others not present in training for a total of 52 unique objects and 52 unique properties. None of the combinations presented in testing were repeated from training. Compounds were presented in a way identical to training, but after each trial, participant were asked to rate their well-formedness on a $1-5$ scale $(1=$ poorly-formed, $5=$ well-formed $)$. Testing blocks exposed participants to three supertypes of testing items: (1) consistent with training, (2) inconsistent with training, and (3) critical.

The consistent items displayed the processes applying independently and not in critical settings, and thus we expected participants from both groups to rate these high. The inconsistent items showed processes failing to apply in appropriate contexts or applying in inappropriate contexts, thus we expected participants from both groups to rate these items low. The critical items were the $\mathrm{CVJ}+\mathrm{sVC}$ and $\mathrm{CV}_{3}+\mathrm{zVC}$ items, which were withheld from training. The crucial prediction was that each participant group should accept different forms as grammatical compounds in these contexts: the Assimilation group should be more likely than the Labialization group to accept epenthesis (e.g., [lofasol]), while the Labialization group should be more inclined than the Assimilation group to accept a form that has simply undergone the labialization process (e.g. [lofsol]).

Results were analyzed with a linear mixed-effects model, treating RATING as a continuous variable. We included random intercepts for participants and items, and random slopes for participants and items 
for all effects of interest that were manipulated within participants or within items. As inferential statistics, we report for each coefficient of interest the t-value obtained by dividing the coefficient estimate by its standard error. For datasets of this size, the $t$ distribution is well approximated by the normal, and thus we assess significance at the $5 \%$ level in a two-tailed test by judging whether the t-value is greater in absolute value than 1.96 (Baayen, Davidson, \& Bates, 2008).

The ratings for items which were consistent with training are illustrated in (6). As predicted, both groups learned the alternations presented in training. Epenthesis between identical consonants was consistent with both languages, and both groups rated forms showing this process highly (no significant difference between the two groups; $t=0.83$ ). Similarly, the lateralization process was rated highly by both groups, although the Labialization group provided ratings that were significantly higher $(\mathrm{t}=2.60)$. Additionally, each group learned the unique process they were exposed to during training: either place assimilation or labialization. Assimilation was consistent only with the Assimilation Language and was rated significantly higher by that group $(\mathrm{t}=-7.76)$. The opposite was true for the labialization process - it was only consistent with the Labialization Language, and the group trained on that language rated it significantly higher $(\mathrm{t}=10.50)$.

(6)

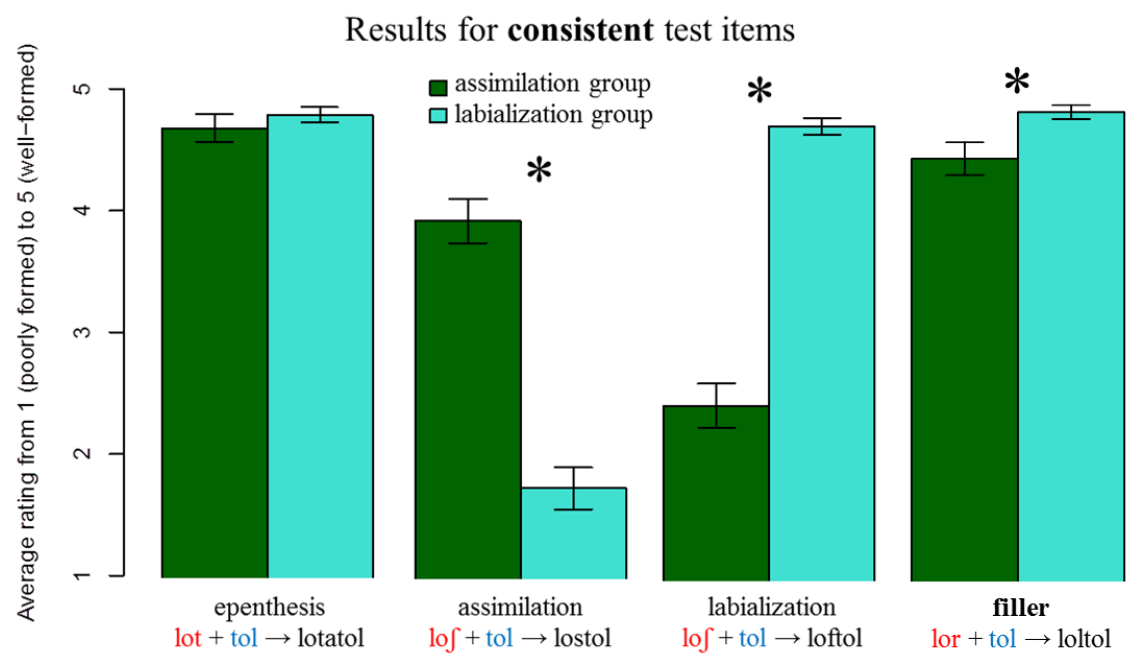

The results for critical items are shown in (7a). We predicted that the critical contexts $\mathrm{CVJ}+\mathrm{sVC}$ and $\mathrm{CV}_{3}+\mathrm{zVC}$ would prompt a differential response pattern from the Assimilation and the Labialization groups: epenthesis rated higher by the Assimilation group than by the Labialization group, and labialization rated higher by the Labialization group than by the Assimilation group. These predictions were borne out, as indicated by a significant interaction between GROUP and ALTERNATION ( $t=7.11)$. Pairwise comparisons performed separately for each alternation revealed higher ratings of epenthesis by the Assimilation group than by the Labialization group $(\mathrm{t}=-2.60)$, and higher ratings of labialization by the Labialization group than by the Assimilation group ( $\mathrm{t}=9.81)$. Additionally, the Assimilation group was not simply more inclined to accept epenthesis across the board. The Assimilation group's ratings of critical epenthesis were significantly higher than their ratings of epenthesis in inconsistent contexts (e.g., $/ \mathrm{lom} /+/$ tol $/ \rightarrow$ [lomatol]; $t=-3.54)$, whereas the Labialization group's ratings were not $(t=-0.19)$, as shown in (7b). Altogether, these results suggest that the Assimilation group inferred the interaction between assimilation and epenthesis from exposure to each process in independent contexts. 
(7)

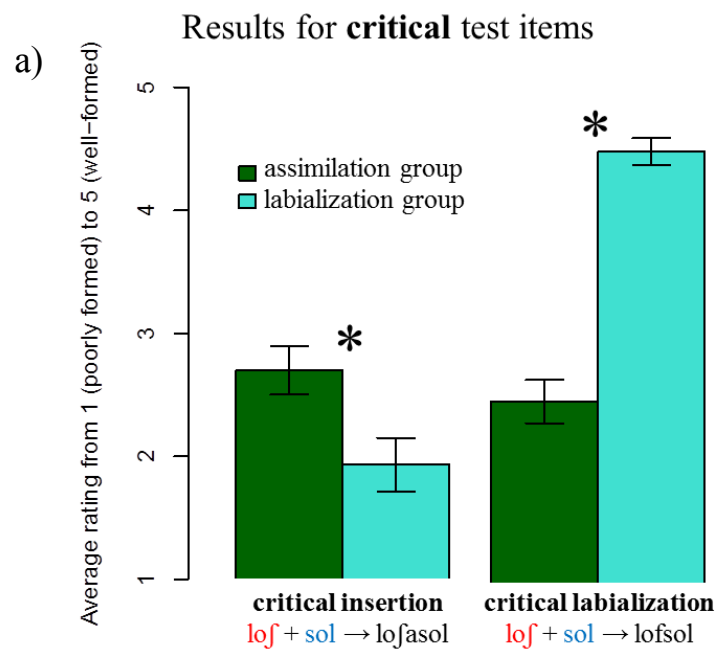

b)

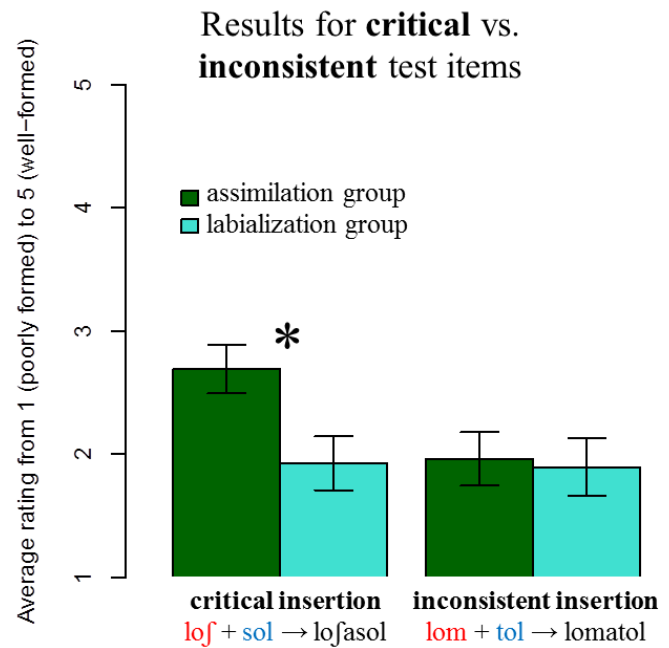

Interestingly, the Assimilation group's ratings assigned to critical epenthesis and critical labialization trials were not significantly different from one another $(\mathrm{t}=0.86)$, and the critical labialization ratings were significantly higher than their ratings of inconsistent changes $(t=-2.71)$. A potential explanation for this result is that the Assimilation group may not have been learning to avoid identity exclusively via epenthesis. Labialization is equally effective at preventing a sequence of identical consonants, as would be many other untested processes. Thus, it could be the case that participants in the Assimilation group learned a more general principle: to avoid identity by any means.

3. Conclusion. We sought to address the question of whether direct evidence of an interaction between phonological processes is necessary for the learning of said interaction. We trained participants on an artificial language in which they observed two processes acting independently and were tested on cases in which the two could interact. Our results suggest that learners are able to use indirect evidence to infer an interaction between phonological processes: participants accepted a familiar repair for novel forms that they induced to be ungrammatical. However, direct evidence may still be needed for learners to acquire the specific ways in which a language repairs illegal forms, as our participants were inclined to also accept repairs for which they had not seen any evidence in training, as long as clearly ungrammatical forms were avoided.

\section{References cited}

Baayen, R. H., D. J. Davidson, \& D. M. Bates. 2008. Mixed-effects modeling with crossed random effects for subjects and items. Journal of Memory and Language 59, 390-412.

Baković, E. 2005. Antigemination, assimilation, and the determination of identity. Phonology 22, 279315. 